\title{
Clinical feasibility of endoscopic submucosal dissection with minimum lateral margin of superficial esophageal squamous cell carcinoma
}

\section{(ㄷ)(1) $(2) \Theta$}

\author{
Authors \\ Hiroki Kuwabara1,2, Seiichiro Abe ${ }^{1}$, Satoru Nonaka', Haruhisa Suzuki ${ }^{1}$, Shigetaka Yoshinaga' ${ }^{1}$ Ichiro Oda', Yutaka \\ Saito ${ }^{1}$
}

Institutions

1 National Cancer Center Hospital, Endoscopy Division, Tokyo, Japan

2 Omori Red Cross Hospital, Department of Gastroenterology, Tokyo, Japan

submitted 1.6.2018

accepted after revision 26.11.2018

\author{
Bibliography \\ DOI https://doi.org/10.1055/a-0838-5064 | \\ Endoscopy International Open 2019; 07: E396-E402 \\ (c) Georg Thieme Verlag KG Stuttgart · New York \\ ISSN 2364-3722
}

Corresponding author

Seiichiro Abe, MD, PhD, Endoscopy Division, National

Cancer Center Hospital, 5-1-1 Tsukiji, Chuo-ku, Tokyo 1040045, Japan

Fax: $+81-3-35423815$

seabe@ncc.go.jp

\section{ABSTRACT}

Background Esophageal stricture following endoscopic submucosal dissection (ESD) for superficial esophageal squamous cell cancer (SESCC) has been associated with wide mucosal defects greater than three-quarters of the luminal circumference. Some patients developed dysphagia and required repeated endoscopic balloon dilation even after steroid therapy. As iodine staining clearly visualized the margin of SESCC, we made a longitudinal mucosal incision close to the margin of the lesion to avoid a mucosal defect involving over three-quarters of the luminal circumference for large lesions. This retrospective study aimed to clarify the clinical feasibility of ESD with minimum lateral margin of SESCC.

Patients and methods Between 2005 and 2013, 94 patients with 94 initial SESCCs had lesions greater than half of the luminal circumference. Of those, 70 patients with 70 SESCCs had achieved endoscopic clearance for the initial SESCC. In this study, endoscopic clearance was defined as en bloc resection of SESCC histologically confined to the mucosa without lymphovascular invasion and with a free deep margin regardless of the lateral margin. This study evaluated the short- and long-term outcomes in patients undergoing endoscopic clearance.

Results In total, 61.4\% (43/70) of the patients had mucosal defects involving over three-quarters of the luminal circumference and $38.5 \%(27 / 70)$ had a positive or indeterminate lateral margin. However, there was no local or nodal recurrence during the median follow-up period of 3.8 years; the 3 -year overall survival rate was $98.5 \%$ and the 3 -year disease-free survival rate was $100 \%$.

Conclusion Using our institutions' strategy, ESD for SESCCs with minimum lateral margins was oncologically acceptable; this approach could reduce the known risk factor of post-ESD stricture.

\section{Introduction}

Endoscopic resection (ER) is accepted as an effective, minimally invasive treatment for early superficial esophageal squamous cell cancer (SESCC) [1-4]. Although extensive ER has become more common as endoscopic techniques by piecemeal endoscopic mucosal resection (EMR) have developed, piecemeal resection is a significant risk factor for local recurrence after ER $[5,6]$. Endoscopic submucosal dissection (ESD) has advanced for en bloc resection regardless of tumor size or tumor location
[7]. Furthermore, ESD for SESCC yields favorable long-term outcomes $[8,9]$.

However, when resecting large lesions, the frequency of esophageal strictures after ESD has increased [10,11]. Esophageal strictures following ER are associated with wide mucosal defects greater than three-quarters of the luminal circumference [12]. When the mucosal defect exceeds three-quarters of the luminal circumference, stricture occurrence is prevalent in $68-100 \%$ cases [ $10-13$ ]. Although oral prednisolone or locoregional steroid injections have shown promising results for the 

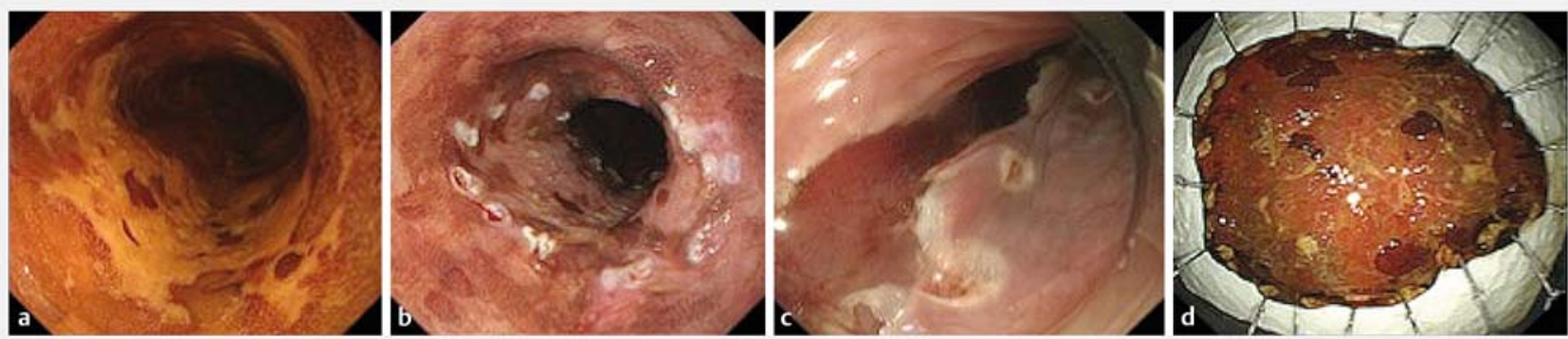

- Fig. 1 Endoscopic submucosal dissection (ESD) strategy for esophageal lesions with minimum lateral margins. a Chromoendoscopy with iodine staining clearly visualized a superficial esophageal squamous cell carcinoma (SESCC). b Markings close to the margins. $\mathbf{c}$ Mucosal incision close to the marking. $\mathbf{d}$ Minimize the luminal circumference for large SESCC to avoid stenosis from excessive resection.

prevention of esophageal strictures following ESD [14-16], some patients may still develop dysphagia and require repeated endoscopic balloon dilation even after steroid therapy [17].

Markings for ESD are generally made $5 \mathrm{~mm}$ away from the borders of the lesion [18]. However, iodine staining enables clear visualization of the SESCC margins [19], so we applied markings with energy devices just on the border and commenced the incision just outside the applied marks so as to minimize luminal defects for large SESCC cases involving over three-quarters of the luminal circumference. This retrospective study aims to clarify the clinical feasibility of ESD with minimum lateral margin of SESCC.

\section{Methods}

\section{Patients}

A total of 268 patients with 289 esophageal lesions consecutively underwent ESD of SESCC at our institution between 2005 and 2013. Endoscopic resection was indicated for histologically proven squamous cell carcinoma on biopsy and was not performed in patients with apparent massive submucosal invasion or nodal metastasis. The depth of invasion was estimated by white-light endoscopy, and magnified narrow-band imaging and chromoendoscopy with iodine staining. Endoscopic ultrasonography was also performed to determine the depth of invasion as necessary. Mizuta et al. [10] reported that a lesion more than half of the luminal circumference was closely associated with an ESD-induced mucosal defect involving over three-quarters of the luminal circumference and was considered to be an independent risk factor for esophageal stricture. Thus, amongst those patients, we investigated those who had a lesion more than half of the luminal circumference and met endoscopic clearance for SESCC and who were followed up without any additional treatment. In this study, endoscopic clearance was defined as en bloc resection of SESCC histologically confined to the mucosa without lymphovascular invasion and with a free deep margin, regardless of the lateral margin. In reference to the Guidelines for Diagnosis and Treatment of Carcinoma of the Esophagus edited by the Japan Esophageal Society, those lesions confined to the epithelium (EP) and lamina propria mucosae (LPM) have almost no risk of metastasis; le- sions limited to the muscularis mucosae (MM) without lymphovascular invasion also have a very low risk of metastasis [20]. At our institution during the study period, a SESCC involving the whole circumference was mainly treated with chemoradiotherapy rather than ESD.

\section{ESD procedure}

The ESD procedure was performed by expert endoscopists skilled in ESD or trainees with support from expert endoscopists. Trainees were defined as those who had limited experience in performing esophageal ESD procedures (30 cases or less), and expert endoscopists had experienced performing more than 30 esophageal ESD procedures [21].

A dual knife (KD-650; Olympus, Tokyo) was used to make markings close to the margins of the lesion. The dual knife and an insulation-tipped (IT) knife nano (KD-612; Olympus) were used for mucosal incision and submucosal dissection. Intraoperative bleeding was treated using a hemostatic forceps (Coagrasper, FD-411 QR; Olympus). ESD was performed with the patient under deep sedation using a combination of midazolam or propofol with pentazocine. The ESD strategy for esophageal lesions with minimum lateral margins comprised these three steps: 1) chromoendoscopy with iodine staining to clearly visualize the margin of SESCC; 2) circumferential marking close to the margins of the lesion, and 3) mucosal incision close to the marking, or on the marking in some cases ( $\bullet$ Fig. $\mathbf{1}$ ). This strategy was applied to lesions more than half of the circumference that were closely associated with a mucosal defect involving over three-quarters of the luminal circumference.

\section{Stricture prevention}

In this study, until 2010, prophylactic endoscopic balloon dilation (EBD) was performed to avoid esophageal stricture following widespread endoscopic resection [22]. Oral prednisolone and/or locoregional triamcinolone injection were introduced in 2011 based on the discretion of the endoscopist.

\section{Histology}

After fixing in $10 \%$ formalin and serial sectioning at 2-mm intervals, the resected specimens were assessed using histological mapping. Expert pathologists were engaged to assess the mac- 
roscopic appearance, tumor size, depth of invasion, lymphatic and vascular involvement, and lateral and vertical margins, with reference to the Japanese Classification of Esophageal Carcinoma $[23,24]$. Lymphovascular involvement was first determined using hematoxylin and eosin (HE)-stained sections; when the diagnosis of lymphovascular involvement was inconclusive using HE-stained sections, immunohistochemistry was also performed with the monoclonal antibody D2-40 and/or Victoria blue or Elastica van Gieson staining.

\section{Follow-up after endoscopic clearance}

All patients who achieved endoscopic clearance generally underwent esophagogastroduodenoscopy (EGD) surveillance on an annual or biannual basis. In addition, a computed tomography $(C T)$ scan was performed every 6 months or 1 year to identify lymph node and distant metastases in patients with muscularis mucosa invasion. A local recurrence was defined as an iodine-unstained area detected adjacent to an ESD scar and cancer cells were verified histologically in a biopsy specimen. Metachronous esophageal squamous cell cancer (ESCC) was defined as ESCC other than local recurrence detected in surveillance EGD.

\section{Assessment}

This study evaluated short- and long-term outcomes in cases with a lesion more than half of the circumference undergoing endoscopic clearance.

In this study, we investigated alcohol consumption, smoking habits, and grade of Lugol-voiding lesion (LVL) as patient background. LVL was graded according to the number of LVLs per endoscopic view (A, no lesions; B, $1-9$ lesions; C, $\geq 10$ lesions) [25].

Technical results, adverse events, and histopathological results were assessed to determine short-term outcomes. Longterm outcomes included local recurrence, lymph node metastasis, distant metastases, 3-year cumulative incidence of metachronous ESCC, 3-year overall survival (OS), and 3-year diseasefree survival (DFS) after endoscopic clearance.

\section{Statistical analysis}

All statistical analyses were performed with EZR (Saitama Medical Center, Jichi Medical University, Saitama, Japan), which is a graphical user interface for R (The R Foundation for Statistical Computing, Vienna, Austria) [26]. Continuous variables were compared using the Mann-Whitney $U$ test and categorical variables were compared using the $x^{2}$ test or Fisher's exact test as appropriate. OS and DFS survival rates were calculated by Kaplan-Meier analysis. OS was measured from the date of ESD to the date of death or the date of the latest confirmation of survival. DFS was measured from the date of ESD to the date of death from ESSC.

\section{Results}

Amongst 268 consecutive patients with 289 SESCCs, 94 patients with 94 initial SESCCs had lesions more than half of the circumference. Of those, 24 patients with 24 SESCCs were ex-

\section{8 concecutive patients with 289 ESCCs \\ - Prior treatment: 14 patients with 35 ESCC \\ - Luminal circumference of the lesion less than 1/2: 160 patients 160 ESCCs \\ 94 patients with 94 ESCCs who treated with minimal lateral margin \\ - SM invasive cancer: 13 patients with 13 ESCC \\ - Mucosal cancer with lymphovascular \\ invasion: 7 patients with 7 ESCC \\ - Additional CRT for mucosal cancer: \\ 4 patients with 4 ESCC \\ 70 patients had achieved endoscopic clearance}

- Fig. 2 In total, 94 patients with 94 superficial esophageal squamous cell carcinoma (SESCCS) had lesions more than half of the circumference. Of those, we investigated 70 patients who met endoscopic clearance for initial SESCC and who were followed up without any additional treatment.

cluded as 13 patients had pathological submucosal invasion, seven patients had mucosal cancer with lymphovascular invasion, and four patients underwent additional chemoradiotherapy for muscularis mucosae lesions. The remaining 70 patients with 70 SESCCs had achieved endoscopic clearance for initial SESCC and were followed up without any additional treatment ( Fig. 2).

The male/female ratio was 6.8:1 (61 men/9 women), and median age was 69 years (range, $50-86$ years); $78.6 \%$ of patients had a current or previous habit of alcohol consumption and $68.6 \%$ of patients were smokers; $88.6 \%$ of patients had multiple dysplastic lesions. More than half of the lesions $(57.1 \%)$ were located in the middle esophagus. The median tumor size was $35 \mathrm{~mm}$ (range, $20-85$ ). With regard to depth of invasion, $4.3 \%$ were EP, $77.1 \%$ were LPM, and $18.6 \%$ of lesions had invaded to the depth of the MM ( $\vee$ Table 1$)$. All lesions had achieved en bloc resection. Complete curative resection with a negative lateral margin was achieved in $61.4 \%$ (43/70) of patients and with a positive lateral margin in $38.6 \%(27 / 70)$ of patients. The median procedure time was 120 minutes (range, 50 -570). The stricture rate was $35.7 \%$ (25/70). Twelve cases were orally administered prednisolone and five patients underwent locoregional triamcinolone injection; $57.1 \%$ (40/70) of patients had a mucosal defect involving over three-quarters of the luminal circumference. Of those, stenosis was seen in 16 of 25 cases (64\%) in the group who underwent preemptive EBD and in 5 of 15 cases (33\%) in the group who underwent steroid treatment ( $\triangleright$ Table 2 ). In terms of lateral margin, tumor size was significantly larger in the positive lateral margin group than in the negative lateral margin group. However, there was no statistically significant difference between the two groups 
- Table 1 Patients $(n=70)$ and tumor characteristics.

\begin{tabular}{l|c|}
\hline Age, median (range), years & $69(50-86)$ \\
\hline Sex & 61 \\
\hline - Male & 9 \\
\hline - Female & \\
\hline Alcohol consumption status & 15 \\
\hline - Non-drinker & 55 \\
\hline - Drinker & \\
\hline Cigarette smoking status & 22 \\
\hline - Non-smoker & 48 \\
\hline - Current smoker or ex-smoker & \\
\hline Location & 6 \\
\hline - Upper esophagus & 40 \\
\hline - Middle esophagus & 24 \\
\hline - Lower esophagus & 34 \\
\hline Tumor depth & 35 \\
\hline - EP & 29 \\
\hline - LP, epithelium; LPM, lamina propria mucosae; MM, muscularis mucosae; \\
\hline - CVL, Lugol-voiding lesion.
\end{tabular}

in the rate of cases with a circumferential mucosal defect greater than three-quarters of the circumference ( $>$ Table 3 ).

During a median follow-up period of 3.8 years, there was no local or nodal recurrence in the 70 patients. The cumulative incidence of metachronous ESCC at 3 years was $11.8 \%$ ( $\triangleright$ Fig.3). LVL grade was associated with progressive increases in the 3-year cumulative incidence of metachronous ESCCs $(0.0 \%, 6.6 \%$, and $23.4 \%$, respectively) ( $\mathbf{F i g} .4)$. The 3 -year OS rate was $98.5 \%$ ( $\mathbf{F i g . 5}$ ) and the 3 -year DFS rate was $100 \%$, respectively.
- Table 2 Short-term outcomes for patients in this study $(n=70)$.

En bloc resection, \%

100

Lateral margin

- Negative 43

- Positive or indeterminate 27

Procedure time, median (range), min

$120(50-570)$

Endoscopist

- Expert 55

- Trainee 15

Mucosal defect greater than $3 / 4$ of luminal circumference, \%

Resection size, median (range), $\mathrm{mm}$

$57.1(40 / 70)$

Steroid treatment

Steroid treatment

- Not used 53

- Oral prednisolone

- Triamcinolone injection 12

Stricture, \% $35.7(25 / 70)$

Perforation, \% $2.9(2 / 70)$

\section{Discussion}

Chromoendoscopy with iodine staining has been reported to be useful in identifying the margins of SESCC $[19,27]$. It allows us to make markings close to the margins of the lesion so as to avoid excessive resection. The strength of this study was that no patient developed local recurrence or nodal metastasis in ESD with minimum lateral margins, and this suggested the SESCCS that achieved endoscopic clearance could be clinically and oncologically followed as $\mathrm{R} 0$ resections.

Esophageal stricture following ESD has been associated with wide mucosal defects greater than three-quarters of the luminal circumference [12]. Mizuta et al. reported that many cases of lesions greater than half of the circumference were the result of a mucosal defect involving over three-quarters of the luminal circumference [10]. Because the minimum lateral margin strategy is a method of reducing the risk of mucosal defects involving over three-quarters of the luminal circumference, we included patients who had a lesion involving more than half of the luminal circumference. This study demonstrated that mucosal defects involving over three-quarters of the luminal circumference were seen in only $57.1 \%$ of cases that had a lesion

- Table 3 Comparison of cases with a negative lateral margin vs positive or indeterminate lateral margin.

\begin{tabular}{|c|c|c|c|}
\hline & Negative lateral margin & Positive or indeterminate lateral margin & $P$ value \\
\hline $\begin{array}{l}\text { Rate of cases with a mucosal defect greater } \\
\text { than } 3 / 4 \text { of the luminal circumference, } \%\end{array}$ & $51.2(21 / 41)$ & $65.5(19 / 29)$ & 0.3 \\
\hline Tumor size, median (range), mm & $35(20-55)$ & $50(20-85)$ & $<0.01$ \\
\hline
\end{tabular}




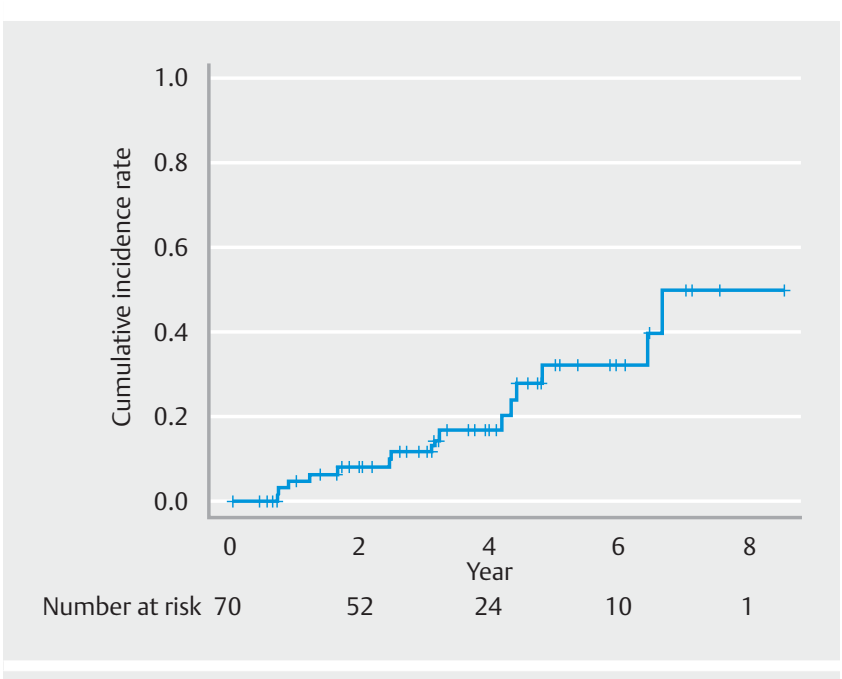

- Fig. 3 Cumulative incidence of metachronous esophageal squamous cell carcinoma (ESSC) in all patients.

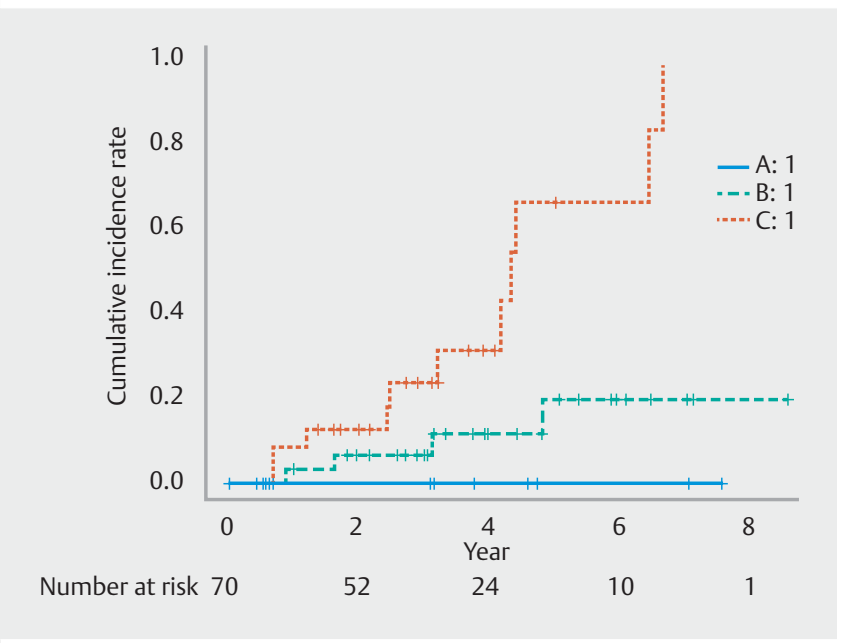

- Fig. 4 Cumulative incidence of metachronous esophageal squamous cell carcinoma (ESSC) according to Lugol-voiding lesion (LVL) grade. LVL was graded according to the number of LVLs per endoscopic view (A, no lesions; B, 1 - 9 lesions; C, $\geq 10$ lesions).

greater than half of the circumference. The proportion of cases with a mucosal defect involving over three-quarters of the luminal circumference was lower than that in standard esophageal ESD [10].

Locoregional steroid injection and oral prednisolone could reduce the stenosis rate of lesions with wide mucosal defects greater than three-quarters of the luminal circumference to $10.0-62.5 \%$ and $5.3-50 \%$, respectively $[14-16,28]$. Steroid treatments were demonstrated to have a significant effect in preventing stricture. However, locoregional steroid injection can cause delayed perforation, and oral prednisolone can cause systemic adverse effects, including diabetes, peptic ulceration, osteoporosis, and increased susceptibility to infection $[29,30]$. Furthermore, some patients with wide mucosal defects greater than three-quarters of the luminal circumference failed to avoid esophageal stricture even with steroid treatment, parti-

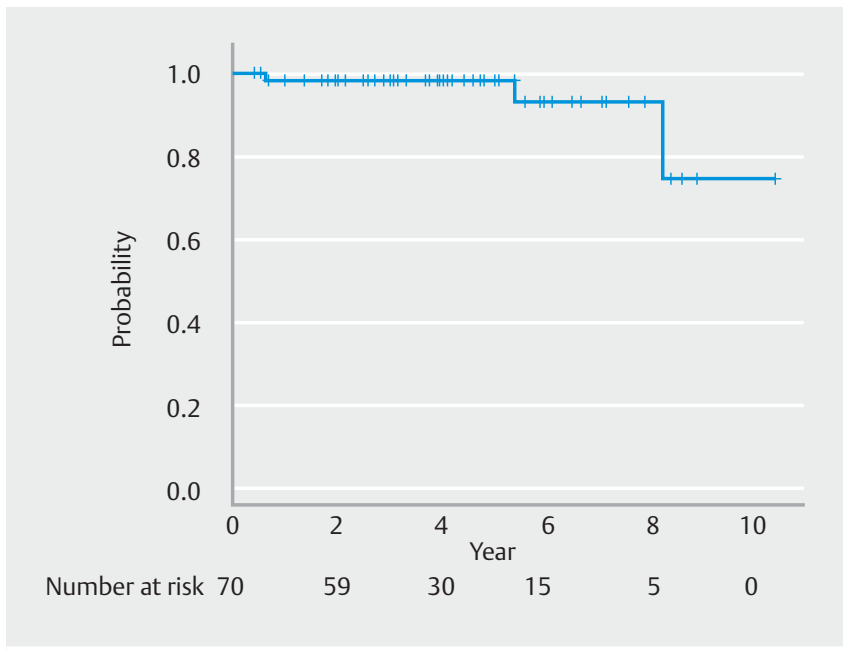

- Fig. 5 Kaplan-Meier curve of the overall survival rate after endoscopic submucosal dissection (ESD).

cularly in those with mucosal defects involving the whole circumference [31]. Therefore, it is important to reduce lesions with a mucosal defect involving over three-quarters of the luminal circumference as much as possible using the minimum lateral margin strategy. We believe our strategy could reduce the risk of extensive ESD and thus minimize preventive steroid administration, which was generally unnecessary for lesions which did not have the risk factor of post-ESD stricture.

En bloc curative resection is considered to be the ideal for both EMR and ESD treatment because it provides an accurate histological assessment and reduces the risk of local recurrence [9]. Ishihara et al. evaluated factors associated with local recurrence following endoscopic resection of SESCC and concluded that multiple piecemeal resection (more than five pieces) was a significant risk factor for local recurrence and that there were few recurrences if en bloc resection could be achieved [6]. In terms of lateral margin, a positive lateral margin tended to occur in large lesions. Although the positive lateral margin rate was $38.6 \%$ in this study, there was no local recurrence in those who achieved endoscopic clearance. Nagami et al. also reported that the rate of en bloc resection with tumor-free margins was $90.4 \%$ and no local recurrence was encountered regardless of tumor margins in ESD [32]. The results of previous reports support the concept of endoscopic clearance but did not support piecemeal resection.

Similar conclusions have been reported in our institution for large colorectal tumors [33]. Electrocautery devices might damage the squamous epithelium on the lateral margin of the lesion, and therefore the pathologists might be unable to evaluate whether or not the margins of the lesion were neoplastic. Since squamous cell carcinoma has a clear boundary with iodine staining, the potential for neoplastic tissue to remain within the lumen is believed to be very low. Furthermore, Matsuda et al. reported squamous cell carcinoma invading under the epithelium in only $0.67 \%$ of surgically excised specimens [34]. There were very few cases of squamous cell carcinomas invading un- 
der the epithelium and this result also supported our strategy of minimum lateral margins for SESCC.

The cumulative recurrence rate was consistent with previous reports, which showed that multiple dysplastic lesions in the esophagus increase the risk of multiple ESCCs [25]. Surveillance endoscopy should be performed focusing on the early detection of metachronous SESCC rather than local recurrence after endoscopic clearance.

This study had some limitations. First, this was a single center, retrospective study. Second, it is difficult to conclude that the endoscopic clearance strategy reported in this study directly resulted in the prevention of stricture formation. Prophylactic steroid treatment was introduced in 2011 to avoid post-ESD esophageal stricture and it became the current standard of care for wide mucosal defects that were greater than three-quarters of the luminal circumference. The steroid treatment could contribute to stricture prevention in our study. Further prospective study is warranted to investigate the efficacy of ESD with minimum lateral margins.

In conclusion, ESD for SESCCs with minimum lateral margins using our institutions' strategy was oncologically acceptable and this approach could reduce the known risk factor of postESD stricture.

\section{Acknowledgments}

The research team would like to thank Mr Saswata Banerjee (Consultant Colorectal Surgeon, Barking, Havering and Redbridge University Hospitals NHS Trust, London, UK) for his kind support for this article. In addition, part of this work was supported by the National Cancer Center Research and Development Fund (25-A-12, 28-K-1, and 29-A-12).

\section{Competing interests}

None

\section{References}

[1] Shimizu Y, Takahashi M, Yoshida T et al. Endoscopic resection is the criterion standard of treatment for patients with early squamous cell neoplasia of the esophagus. Gastrointest Endosc 2012; 76: 464-465

[2] Inoue $\mathrm{H}$, Tani M, Nagai K et al. Treatment of esophageal and gastric tumors. Endoscopy 1999; 31: 47 - 55

[3] di Pietro M, Canto MI, Fitzgerald RC. Endoscopic management of early adenocarcinoma and squamous cell carcinoma of the esophagus: Screening, diagnosis, and therapy. Gastroenterology 2018; 154: $421-436$

[4] Katada C, Muto M, Momma K et al. Clinical outcome after endoscopic mucosal resection for esophageal squamous cell carcinoma invading the muscularis mucosae - a multicenter retrospective cohort study. Endoscopy 2007; 39: 779-783

[5] Katada C, Muto M, Manabe T et al. Local recurrence of squamous-cell carcinoma of the esophagus after EMR. Gastrointest Endosc 2005; 61: $219-225$
[6] Ishihara R, lishi H, Takeuchi Y et al. Local recurrence of large squamous-cell carcinoma of the esophagus after endoscopic resection. Gastrointest Endosc 2007; 67: 799-804

[7] Ishihara R, lishi H, Uedo $\mathrm{N}$ et al. Comparison of EMR and endoscopic submucosal dissection for en bloc resection of early esophageal cancers in Japan. Gastrointest Endosc 2008; 68: 1066-1072

[8] Ono S, Fujishiro M, Niimi K et al. Long-term outcomes of endoscopic submucosal dissection for superficial esophageal squamous cell neoplasms. Gastrointest Endosc 2009; 70: 860-866

[9] Takahashi H, Arimura Y, Masao H et al. Endoscopic submucosal dissection is superior to conventional endoscopic resection as a curative treatment for early squamous cell carcinoma of the esophagus (with video). Gastrointest Endosc 2010; 72: 255-264

[10] Mizuta H, Nishimori I, Kuratani Y et al. Predictive factors for esophageal stenosis after endoscopic submucosal dissection for superficial esophageal cancer. Dis Esophagus 2009; 22: 626-631

[11] Ono S, Fujishiro M, Niimi K et al. Predictors of postoperative stricture after esophageal endoscopic submucosal dissection for superficial squamous cell neoplasms. Endoscopy 2009; 41: 661-665

[12] Katada C, Muto M, Manabe T et al. Esophageal stenosis after endoscopic mucosal resection of superficial esophageal lesions. Gastrointest Endosc 2003; 57: 165-169

[13] Shi Q, Ju H, Yao L-Q et al. Risk factors for postoperative stricture after endoscopic submucosal dissection for superficial esophageal carcinoma. Endoscopy 2014; 46: 640-644

[14] Yamaguchi N, Isomoto H, Nakayama T et al. Usefulness of oral prednisolone in the treatment of esophageal stricture after endoscopic submucosal dissection for superficial esophageal squamous cell carcinoma. Gastrointest Endosc 2011; 73: 1115-1121

[15] Hashimoto S, Kobayashi M, Takeuchi M et al. The efficacy of endoscopic triamcinolone injection for the prevention of esophageal stricture after endoscopic submucosal dissection. Gastrointest Endosc 2011; 74: 1389-1393

[16] Hanaoka N, Ishihara R, Takeuchi Y et al. Intralesional steroid injection to prevent stricture after endoscopic submucosal dissection for esophageal cancer: a controlled prospective study. Endoscopy 2012; 44: 1007 - 1011

[17] Hanaoka N, Ishihara R, Uedo $\mathrm{N}$ et al. Refractory strictures despite steroid injection after esophageal endoscopic resection. Endosc Int Open 2016; 4: E354-359

[18] Bhatt A, Abe S, Kumaravel A et al. Indications and techniques for endoscopic submucosal dissection. Am J Gastroenterol 2015; 110 : $784-791$

[19] Shiozaki H, Tahara H, Kobayashi K et al. Endoscopic screening of early esophageal cancer with the Lugol dye method in patients with head and neck cancers. Cancer 1990; 66: 2068-2071

[20] Kuwano H, Nishimura Y, Oyama T et al. Guidelines for Diagnosis and Treatment of Carcinoma of the Esophagus April 2012 edited by the Japan Esophageal Society. Esophagus 2015; 12: 1-30

[21] Ohata K, Fu K, Sakai E et al. Esophageal endoscopic submucosal dissection assisted by an overtube with a traction forceps: An animal study. Gastroenterol Res Pract 2016; 2016: 3186168

[22] Ezoe Y, Muto M, Horimatsu T et al. Efficacy of preventive endoscopic balloon dilation for esophageal stricture after endoscopic resection. J Clin Gastroenterol 2011; 45: 222-227

[23] Japan Esophageal Society. Japanese Classification of Esophageal Cancer, 11th Edition: part I. Esophagus 2017; 14: 1-36

[24] Japan Esophageal Society. Japanese Classification of Esophageal Cancer, 11th Edition: part II and III. Esophagus 2017; 14: 37 - 65

[25] Katada C, Yokoyama T, Yano T et al. Alcohol consumption and multiple dysplastic lesions increase risk of squamous cell carcinoma in the esophagus, head, and neck. Gastroenterology 2016; 151: 860-867 
[26] Kanda Y. Investigation of the freely available easy-to-use software "EZR" for medical statistics. Bone Marrow Transplant 2013; 48: 452 458

[27] Ban S, Toyonaga A, Harada $\mathrm{H}$ et al. lodine staining for early endoscopic detection of esophageal cancer in alcoholics. Endoscopy 1998; 30: $253-257$

[28] Abe S, lyer PG, Oda I et al. Approaches for stricture prevention after esophageal endoscopic resection. Gastrointest Endosc 2017; 86: $779-791$

[29] Matsuda Y, Kataoka N, Yamaguchi T et al. Delayed esophageal perforation occurring with endoscopic submucosal dissection: A report of two cases. World J Gastrointest Surg 2015; 7: 123-127

[30] Ishida T, Morita Y, Hoshi N et al. Disseminated nocardiosis during systemic steroid therapy for the prevention of esophageal stricture after endoscopic submucosal dissection. Dig Endosc 2015; 27 : 388 391

[31] Kadota T, Yano T, Kato T et al. Prophylactic steroid administration for strictures after endoscopic resection of large superficial esophageal squamous cell carcinoma. Endosc Int Open 2016; 4: E1267-E1274

[32] Nagami $Y$, Ominami M, Shiba $M$ et al. The five-year survival rate after endoscopic submucosal dissection for superficial esophageal squamous cell neoplasia. Dig Liver Dis 2017; 49: 427-433

[33] Makazu M, Sakamoto T, So E et al. Relationship between indeterminate or positive lateral margin and local recurrence after endoscopic resection of colorectal polyps. Endosc Int Open 2015; 3: 252 - 257

[34] Matsuda K, Watanabe H, Kuwabara S et al. Esophageal submucosal tumor (SMT)-like carcinoma [Japanese with English abstract]. Stomach Intestine 1997; 32: 671 -689 